\title{
Análisis de las políticas públicas en la práctica: El control de gestión en las Administraciones regionales
}

SUMARIO: 1. PREMISA. 2. LAS CULTURAS ORGANIZATIVAS DE LA ADMINISTRACION PUBLICA. 3. ANALISIS FINANCIERO Y ANALISIS DE COSTES. 4. ANALISIS DE LAS POLITICAS PUBLICAS. 5. EL ITINERARIO DE ANALISIS. 6. PROBLEMAS ORGANIZATIVOS DEL CONTROL DE GESTION.

\section{PREMISA}

El objetivo de este capítulo consiste, por así decirlo, en pasar de la teoría a la práctica demostrando cómo pueden ser utilizados los conceptos básicos del análisis de las políticás públicas. Tomaré como modelo un ejemplo, el de la introducción del control de la gestión en la Administración pública italiana, sobre todo a nivel regional, para tratar de aclarar los posibles objetivos y poner de relieve los principales problemas que debemos afrontar probablemente en el momento de ponerlos en práctica.

Para ello se necesitan dos premisas.

La primera afecta al significado mismo de la palabra control, y trata de distinguir entre el significado francés y el anglosajón de dicho término. Como es sabido, la palabra control viene del francés contre-rôle, y significa sustancialmente verificación, examen de que lo que se ha realizado era conforme a lo previsto anteriormente. Ello implica necesariamente que el controlador sea un sujeto diverso del controlado, y aunque en teoría, sería posible imaginar un control-verificación no es- 
trictamente legal, de hecho el término ha acabado identificándose con el control de legitimidad y de oportunidad sobre los actos.

El segundo significado, el anglosajón, al que nos referimos cuando hablamos de control de gestión, es totalmente distinto. Si se me permite una cita extraña a la materia, en los primeros libros de espionaje de John LE CARRÉ aparecía este personaje cuyo nombre era precisamente Control, el jefe absoluto del servicio. Es evidente que su labor no era la de verificar el comportamiento de los agentes, sino, ante todo, la dirección y orientación de su actividad. Y es en este sentido de actividad exquisitamente de dirección como el control de gestión se ha desarrollado en las empresas privadas. Así, por ejemplo, en una publicación de una de las mayores sociedades mundiales de revisión de balances, la Arthur Andersen, encontramos una cita de este tenor: «los controles internos son todos métodos a través de los cuales una organización gobierna sus actividades para conseguir su objetivo».

El punto de partida de estas reflexiones consiste precisamente en entender claramente que cuando hablamos de gestión en la Administración pública presuponemos la adopción del segundo significado del término. Organizar un sistema de control no sirve tanto para decir si el sujeto $\mathrm{X}$ o la unidad organizativa $\mathrm{Y}$ se han comportado bien o mal, sino, ante todo, para tratar de clarificar lo que habrá que hacer mañana. Es preciso eliminar cualquier duda a este respecto.

La segunda premisa necesaria se refiere a lo que podemos esperar de una actividad de control de gestión, y en particular intenta poner en guardia contra una actitud que hace de dicho instrumento la panacea para todos los males que afectan a la Administración pública italiana. De ninguna manera debemos pensar que ésta pueda o deba sustituir una serie de actividades directamente ligadas en la práctica. Así, el control de gestión se relaciona con la actividad de programación (la fijación de los objetivos y la liberación de los recursos necesarios para alcanzarlos), con la determinación de la productividad del trabajo (al objeto, por ejemplo, de distribuir los incentivos monetarios), con los procedimientos de elección racional de las inversiones a efectuar (del tipo Fondo de Inversiones para la Ocupación), y, en definitiva, también con actividades de control-verificación (por ejemplo, el juicio de corrección contable del Tribunal de Cuentas). Qué duda cabe que entre todas estas actividades y el control de gestión deberían desarrollarse sinergias significativas (en el sentido de que pueden intercambiarse una serie de inputs relevantes), pero sería profundamente erróneo imaginar que los objetivos son intercambiables y que, por consiguiente, una de estas operaciones puede reabsorber completamente las demás. $\mathrm{Ni}$ la actividad de programación puede hacer inútil el control de gestión, ni viceversa. A este último le corresponde la detección de los objetivos y recursos de la acción administrativa. Para entender este pun- 
to conviene introducir el concepto de cultura organizativa, entendiendo por ello el conjunto de valores generalmente compartidos por los miembros de la Administración y, en cierto modo, encarnados por los mecanismos organizativos y procedimentales.

\section{LAS CULTURAS ORGANIZATIVAS DE LA ADMINISTRACION PUBLICA}

Es indudable que la cultura tradicional de la Administración pública italiana se ha basado, durante largo tiempo, en el concepto de control-verificación, en el sentido ya descrito. Esta ha inspirado los controles de legitimidad cuya inutilidad, y en ciertos aspectos perniciosidad, ya se han comentado. La idea de determinar para cada acto administrativo, ante todo, su correspondencia con un conjunto de reglas formales a menudo escasamente o nada relacionadas con los objetivos de la acción administrativa, centrados sobre todo en el respeto de los mecanismos procedimentales, quizá convenía en un período en el que las actividades eran de tipo exclusivamente regulativo. Sin embargo, carece de valor en el desarrollo de los servicios públicos y, más en general, en el Estado de bienestar. Por otra parte, a lo largo del tiempo esta cultura del control se ha identificado en una serie de sectores sin justificación alguna, por lo que debe ser decididamente superada si pretendemos mejorar la eficacia y la eficiencia de la Administración pública.

A la cultura tradicional vino a añadirse, a lo largo de los años sesenta y setenta, la llamada cultura de la programación. Se necesitaría mucho tiempo para explicar lo que se entendía efectivamente con dicho término, pero, en general, podemos afirmar que ésta se identificaba con el intento de predeterminación estrictamente racional de las acciones a emprender. Como ya se ha dicho, la programación es un aspecto esencial de las Administraciones públicas contemporáneas. Sin embargo, refiriéndonos a la cultura que la ha inspirado, debemos subrayar dos peligros. El primero consiste en la asunción acrítica del llamado ideal racional sinóptico, según el cual es necesario definir un cuadro orgánico completo y coherente de todos los objetivos, los instrumentos y las relaciones para reconstruir los costes y los beneficios de cada alternativa, al objeto de minimizar los primeros y maximizar los segundos. Como sabemos, este ideal se ha encarnado en mecanismos organizativos complejos (de programación en cascada, ascendentes, etcétera) que no han funcionado, no sólo por falta de voluntad, por la oposición de intereses constituidos o por falta de preparación, sino también por el simple hecho de que es imposible controlar una tal complejidad, ya que no disponemos de instrumentos analíticos adecuados 
al delirio de omnipotencia que este enfoque (y esta ideología) implicaba.

El segundo peligro se halla directamente relacionado con el primero. En efecto, aun cuando se asumía correctamente un enfoque sectorial, la cultura de la programación tendía a predeterminar, más o menos rígidamente, las características de las acciones sucesivas a través del establecimiento de estándares, lo que sería perfectamente aceptable si después no se cometieran errores conceptuales, incluso graves, en su utilización. Un ejemplo clásico procede de los mecanismos de la programación sanitaria en lo que respecta a la búsqueda de eficiencia en la utilización de las estructuras hospitalarias. Se partía de la consideración obvia de que maximizando el uso de los instrumentos de producción (en este caso las camas hospitalarias) se habrían podido conseguir ahorros significativos. De ahí la construcción de indicadores -ocupación de las camas, estancia media, índice de rotación-que tenían por objeto el control de dicha dimensión. Lo malo es que después se corría el riesgo de confundir los indicadores con las variables, considerando que la eficiencia (es decir, la relación entre costes y productos), e incluso la eficacia (es decir, la relación entre objetivos y resultados), consistían, en efecto, en el hecho de que el valor observado se correspondiera con el estándar predeterminado. Dicha actitud, que podemos calificar de fetichismo del estándar, era y es peculiar porque cerraba la puerta a cualquier indagación sobre el funcionamiento efectivo de los servicios e imaginaba que éste era el único camino posible para obtener el mismo grado de eficiencia y eficacia.

Por supuesto, la superación de las insuficiencias propias de la cultura de la programación no implica echarlo todo por la borda. Estas observaciones, sobre las cuales volveremos más adelante, no deben ser interpretadas como un ataque a la actividad planificadora que, al contrario, por múltiples motivos que no podemos tratar aquí, habría que desarrollar y hacer más eficaz.

Sin embargo, existe un tercer tipo de cultura organizativa que, a pesar de encontrarse todavía ausente de la Administración pública italiana, empieza a presentarse como una alternativa significativa a las precedentes. Me refiero a la cultura de la revisión que se ha desarrollado en el sector privado a partir de la actividad de certificación presupuestaria. Como es sabido, se trata de una técnica que empieza a utilizarse entre nosotros en la medida en que la certificación se ha hecho obligatoria para las sociedades anónimas cotizadas en bolsa y para las sociedades con participación estatal. Esta sirve, sobre todo, para garantizar no sólo que no se produzcan manipulaciones o falsificaciones en la compilación de los documentos contables, sino, sobre todo, que el valor patrimonial y económico general de la empresa resultante de los libros contables sea el verdadero. Esta operación reviste un interés evi- 
dente en un mundo como el actual en el que la propiedad de la empresa se halla fraccionada entre una pluralidad de accionistas que no disponen de poderes significativos de dirección y gestión diaria. En cambio, resulta más complejo comprender la utilidad de un ejercicio de este tipo aplicado a la Administración pública y, en particular, a la Administración pública local. Ello no significa que un método de análisis de la contabilidad tendente a evitar sorpresas desagradables (la existencia de un déficit oculto, por ejemplo) no pueda ser especialmente relevante, y sería positivo que la actividad del Tribunal de Cuentas se desarrollara en este sentido. Conviene igualmente evitar la repetición de los errores del pasado, creyendo que basta con sustituir el análisis contable por el de legitimidad para que se resuelvan automáticamente todos los problemas de eficiencia, eficacia y equidad de la acción administrativa.

A estos tres tipos de cultura quisiera añadir otro, correspondiente al instrumento al que nos estamos refiriendo, es decir, al control de gestión: la cultura de la valoración. Quizá la tarea más urgente para una Administración como la actual, que suministra una gran cantidad de servicios y que, por motivos exógenos, en el futuro no parece destinada a una expansión comparable a la de épocas anteriores, consiste en interrogarse de forma atenta y continuada sobre lo que está sucediendo para saber si las acciones que realiza son útiles y si éstas se desarrollan del mejor modo posible. Se trata de un elemento prácticamente ausente no sólo de la cultura política (debido a su naturaleza, más orientada a la prospectiva y al proyecto), sino también de la cultura estrictamente administrativa, más acostumbrada a contemplar los problemas en clave organizativa y de gestión. A pesar de ello, aunque sólo sea de sentido común valorar hasta qué punto se ha llegado a la satisfacción de determinadas demandas o necesidades, constituye una exigencia ineludible incluso - y sobre todo- en las Administraciones públicas que no disponen del control externo que representa el mercado.

Siendo ésta la cultura que pretendemos introducir sistemáticamente en el seno del proceso político administrativo, podemos definir pues el control de gestión como el instrumento de dirección que produce informaciones basadas en análisis retrospectivos. La segunda parte de la definición se refiere al hecho de que a través del control de gestión nos hallaremos en condiciones de poder saber constantemente, en tiempo real, cómo se desarrollan las actividades y qué resultados se consiguen. Sin embargo, actividad de dirección significa, en primer lugar, que el objeto que se persigue no es meramente cognoscitivo, sino que tiende a producir hipótesis empíricamente verificables relativas a lo que es posible hacer para mejorar la situación existente. Se trata de estudiar el pasado y el presente para producir propuestas para el futuro, 
analizando sistemáticamente la realidad al objeto de verificar si los objetivos, las demandas, las necesidades y los problemas han variado y requieren nuevas respuestas. Este es, a mi entender, el objetivo fundamental al que debemos responder, por lo que podemos pasar ahora a examinar lo que es posible hacer en concreto.

\section{ANALISIS FINANCIERO Y ANALISIS DE COSTES}

Históricamente, las primeras experiencias de introducción del control de gestión en la Administración pública se han concretado esencialmente en el análisis financiero, el primer objeto al que debemos referirnos brevemente.

Una de las polémicas que durante largo tiempo han marcado el debate sobre la eficiencia de la Administración pública se refiere a la cuestión de los residuos pasivos, por lo que las primeras actividades de control de gestión han afectado esencialmente a la determinación de capacidad de gasto.

Este tema constituye, efectivamente, un serio problema para nuestro país si tenemos en cuenta que, en el juicio de igualación del Presupuesto de 1984, el Tribunal de Cuentas constató que la región de Sicilia, ya sea en el sistema bancario, ya en su cuenta de tesorería, disponía de la increíble suma de 6.750 millones de liras frente a un presupuesto total (excluidos los residuos) no superior a los 9.000 millones. Entender las dimensiones de esta notoria incapacidad de ejecutar las decisiones y detectar las partidas en que ésta aparece más grave representa, sin duda, un aspecto significativo e importante cuyo estudio puede ayudar a mejorar efectivamente la decisión política en todas las fases relevantes.

Y ello es así porque el análisis financiero no se limita normalmente a la falta de capacidad de gasto, sino que incluye también otros aspectos significativos. En primer lugar, éste permite igualmente un análisis de los perceptores del gasto que, en el caso de la región, implica no sólo los beneficiarios finales, sino también los numerosos casos de intermediación. De este modo podemos territorializar el gasto, es decir, atribuir a las distintas circunscripciones geográficas la suma que reciben bajo forma de transferencias o de administración directa. Del mismo modo, es posible introducir elementos dinámicos en el análisis, tratando de reconstruir las series históricas o las tendencias del gasto regional.

Todos estos aspectos son del mayor interés y, sin embargo, hay que advertir que si bien es cierto que un sujeto que no utiliza totalmente los recursos puestos a su disposición con toda probabilidad padece problemas organizativos relevantes, también es cierto que no todos los 
modos de gastar los recursos disponibles son correctos. En otras palabras, el análisis de la capacidad de gasto puede ofrecer una serie de informaciones significativas, sobre todo si se desagrega de distintos modos; sin embargo, sólo funciona en sentido negativo, diciéndonos muy poco sobre los factores que determinan eventualmente la acumulación de residuos o sobre la funcionalidad efectiva del gasto realizado. Después de todo, es mejor, sin duda, evitar el gasto a costa de la ineficiencia que utilizar mal los recursos disponibles. Sobre este punto volveremos más adelante.

El segundo tipo de objetivo atribuido al control de gestión, siempre desde el punto de vista histórico, es más sofisticado, ya que se refiere a la puesta a punto de un sistema de contabilidad de costes o contabilidad industrial, como también se le llama a menudo. En sustancia, con este término se pretende indicar la construcción de un esquema analítico en el que sea posible relacionar los recursos utilizados con los bienes efectivamente adquiridos. $\mathrm{El}$ presupuesto resultante, conocido como presupuesto de rendimiento, indicará, no la suma total correspondiente al alquiler de los locales, sino el precio de cada metro cuadrado alquilado; no el costo del mantenimiento del automóvil de representación, sino el costo por cada kilómetro recorrido. El objetivo evidente de un ejercicio de este tipo es el control de los gastos por razones de economía general. Se trata, sin duda, sobre todo en lo relativo a municipios y provincias, de un objetivo significativo e importante; sin embargo, no hay que olvidar tampoco que en el caso de las Administraciones regionales más del 85 por 100 del presupuesto procede de transferencias de otros sujetos en la medida en que la actividad administrativa regional no es y, según la teoría dominante y la propia Constitución, no debe ser una actividad de administración directa, sino que debe realizarse a través de mecanismos de delegación, de financiación directa o de predisposición de incentivos. Por tanto, invertir recursos en la construcción de una contabilidad de costos compleja aparece como algo relevante sólo para lo que podemos considerar como gastos generales de la región, justificándose no tanto en función del posible ahorro, sino, sobre todo, por la exigencia de presentarse ante sus interlocutores, es decir, los perceptores de las transferencias, con los requisitos necesarios para hablar de análisis de eficiencia y eficacia. En cambio, a mi entender, no tiene sentido forzar el instrumento para cubrir otras exigencias y todavía menos este autoanálisis regional debe significar la renuncia a analizar lo que otros sujetos hacen en las materias objeto de financiación regional. Es decir, no basta con que las actividades regionales, en sentido estricto, se desarrollen con el máximo de economía, sino que se trata también de asegurar que la mayoría de los recursos presupuestarios regionales se utilice del modo más eficiente y eficaz. 


\section{EL ANALISIS DE LAS POLITICAS PUBLICAS}

De acuerdo con lo anterior, las experiencias de control de gestión de las administraciones regionales tienen que tomar un camino distinto aunque pueda resultar más difícil y ambicioso. El control de gestión debe convertirse en un instrumento para el análisis retrospectivo, inmediato y continuado de la eficiencia y la eficacia de las políticas públicas. En determinados entes, como los municipios o las unidades sanitarias locales, en los que la producción y distribución directa de bienes y servicios constituye la actividad central, el análisis financiero y, sobre todo, la contabilidad de costes pueden revestir, probablemente, mayor importancia; sin embargo, en el análisis de las regiones deben plantearse problemas de otra naturaleza tomando en consideración las políticas realmente llevadas a cabo (no sólo directamente, sino también por delegación $\mathrm{y} / \mathrm{o}$ mediante transferencias financieras).

$Y$ es precisamente en este punto en el que la revolución intelectual, a la que hacíamos referencia al hablar de la necesidad de introducir la cultura de la valoración, aparece más urgente. Debemos plantearnos, en efecto, una serie de preguntas de carácter general: «¿Para qué sirve la política?»y, por tanto, "¿para qué debería servir?». $\mathrm{Y}$, por otra parte, «¿cuánto cuesta obtener estos resultados?» y, por tanto, «¿se podría gastar menos?». La determinación de la eficacia y la eficiencia de las políticas regionales no puede limitarse al análisis de las acciones directamente desarrolladas por las Consejerías, sino que debe abarcar también el examen de lo que realizan los municipios, las administraciones provinciales, las comunidades de montaña, las unidades sanitarias locales $y$, en general, todos los perceptores de transferencias regionales.

Tradicionalmente, la cultura de la programación ha afrontado esta problemática a través de la fijación de estándares, es decir, de parámetros para medir la correspondencia de las acciones desarrolladas con los objetivos prefijados por el legislativo o el ejecutivo.

El problema reside, en primer lugar, en el hecho de que, a menudo, en la práctica político-administrativa dicha predeterminación de los objetivos se realiza de forma unívoca y coherente, de modo que el examen de la eficiencia y la eficacia no va más allá de la conocida lamentación, según la cual, mientras esto ocurra, la acción pública está condenada a actuar de modo subóptimo. En segundo lugar, sin embargo, hay que reconocer también que la atribución de un valor legal a los estándares fomentada por la cultura de la programación es difícil y peligrosa, puesto que, a menudo, no nos hallamos en condiciones de saber con exactitud cuál es el nivel «justo» de servicio o el costo «justo» que hay que soportar. Por ello, reclamar la fijación de estándares 
rígidos conlleva el peligro de descargar en el legislador, y en general en el sistema político, las insuficiencias culturales del mundo administrativo y de la gestión (incluyendo en este último a los analistas). Ello no significa, obviamente, que dotarse de una legislación menos contradictoria y/o de instrumentos de programación más precisos, y en algunos aspectos más vinculantes, no constituya un objetivo digno de ser perseguido, sino únicamente que la introducción del control de gestión no puede depender de ésta.

La idea esencial es que entre una predeterminación rígida de las acciones y el desinterés total con respecto a los resultados conseguidos (es decir, la situación actual) existe un espacio para obtener mejores resultados (que no quiere decir óptimos).

Esencialmente, el tipo de razonamiento es el siguiente: si bien a menudo nos hallamos en condiciones de determinar con precisión absoluta lo que debería hacerse para maximizar los beneficios de la acción administrativa, minimizando al propio tiempo los costes, lo cierto es que la observación empírica nos permite verificar que, comparativamente, se pueden identificar diferencias significativas de comportamiento, ya sea en relación a los costes o a los resultados entre una unidad productiva y otra (un negociado, un municipio, una unidad sanitaria local), con respecto al suministro de bienes y servicios homogéneos entre sí. Por ello, el control de gestión debe construir un sistema de indicadores capaces de suministrar a los responsables de la producción y la distribución de servicios informaciones significativas relativas a los resultados medios conseguidos y a los costos medios soportados en situaciones comparables. Al mismo tiempo, ello permitirá constituir un sistema de señales de alarma susceptibles de poner en evidencia todas las situaciones en las que el valor del indicador refleja una desviación anormal con respecto a la media. Sin embargo, de estos indicadores no deben deducirse consecuencias automáticas con respecto a la adopción de posibles sanciones, sino únicamente la exigencia de un análisis más profundo para tratar de comprender cuáles son los factores que determinan dicha anormalidad. El siguiente ejemplo evidenciará el peligro implícito en el reconocimiento de un valor legal de estándar a los indicadores de control de gestión.

En 1983, el costo por cada niño usuario de una guardería pública se situaba en Lombardía alrededor de los nueve millones de liras al año, y desde entonces habrá aumentado probablemente. Este dato representa ya un cierto grado de información. Este coste podría considerarse, en efecto, excesivo; después de todo - se dice-, una transferencia de cuatro a cinco millones a cada familia necesitada del servicio podría resolver probablemente el problema de forma satisfactoria para los usuarios, además de suponer un ahorro para el erario público. Sin embargo, esta posición contiene claramente un juicio de va- 
lor sustancialmente político con respecto al equilibrio necesario entre el gasto público y la satisfacción de una serie de objetivos posibles (que no incluyen sólo la custodia de los hijos de las madres trabajadoras, sino también, por ejemplo, la socialización de la primera infancia). Para poder fijar un estándar habría que resolver el difícil teorema de la cumulabilidad de las preferencias individuales. $\mathrm{La}$ cultura de la valoración y del control de gestión procedería, en cambio, de un modo distinto. De hecho, una simple distribución porcentual de los gastos efectivamente realizados en las distintas guarderías municipales de la región revelaría probablemente que la media de nueve millones es la resultante de una situación en la que el 60 por 100 de los casos se distribuyen en torno a la media, con dos extremos en los que los costes giran respectivamente alrededor de los $2 / 3$ millones y $15 / 18$ millones al año. A partir de ahí habría que averiguar cuáles son los factores que determinan estos resultados: donde se gasta poco es posible que el servicio ofrecido sea cualitativamente distinto e inferior al mínimo aceptable. También puede ocurrir que las dimensiones de la unidad productiva, debido a economías o deseconomías de escala relevantes, determinen los costes medios. Por ejemplo, mediante un ulterior estudio, podremos descubrir que en algunas situaciones los costes elevados dependen de la decisión de incorporar un porcentaje importante de niños disminuidos que exigen un cuidado y una atención especial, provocando así el aumento de los gastos de personal. En este caso, la valoración política sería que los gastos adicionales están perfectamente justificados en base al deber de solidaridad social. Es evidente, por tanto, que la adopción de estándares rígidos comportaría el riesgo de determinar una situación en la que los responsables de la producción, por temor a posibles sanciones, renunciarían a enriquecer la calidad del servicio excluyendo, por ejemplo, a los niños disminuidos.

Este ejemplo ilustra claramente lo que debería ser el objetivo del control de gestión y el camino para conseguirlo. No se trata de marcar la dirección de la actuación regional y los procedimientos necesarios para seleccionar las mejores opciones -el verdadero objeto de la programación-, sino de un mecanismo informativo para saber lo que está sucediendo con objeto de mejorar nuestros conocimientos sobre lo que es posible hacer efectivamente para mejorar la eficacia y la eficiencia. Un sistema de indicadores que nos ayude a construir un sistema de señales de alarma capaces, a su vez, de facilitar análisis más pormenorizados para detectar los factores que determinan las diferencias de rendimiento entre cada sector y cada unidad productiva. Y ello con el convencimiento, avalado por numerosas investigaciones realizadas en Italia y fuera de ella, de que es posible identificar dichos factores con un grado suficiente de aproximación, ya que su número no excede - como a menudo se afirma - las posibilidades reales de control, limitándose, por lo general, a algunos elementos sencillos. 


\section{EL ITINERARIO DE ANALISIS}

Después de haber comentado los posibles objetivos de la introducción del control de gestión conviene explicitar ahora el itinerario lógico y analítico necesario para poner en práctica toda la operación. La siguiente figura ofrece un cuadro ilustrativo de las prioridades de análisis.

Como punto de partida diremos que hablar simplemente de análisis de las políticas públicas no basta para resolver el problema del objeto de estudio. Las políticas públicas no existen naturalmente, son el resultado de una reconstrucción realizada por el investigador o el operador. En la vida administrativa diaria nos enfrentamos, en efecto, a una serie de leyes, reglamentos, programas, declaraciones oficiales e incluso preferencias individuales de un responsable político y/o administrativo. La agregación de dichas informaciones en unidades de mayor dimensión, que podemos llamar políticas públicas, es una operación altamente arbitraria en gran medida dependiente de lo que pretendemos explicar efectivamente y del nivel de profundización que deseamos alcanzar (conscientes siempre de la necesidad de elegir entre la exigencia de encuadramiento general y la precisión en la detección de las relaciones causales). Así, la utilización como unidad de análisis de cada capítulo presupuestario no llevará a resultados prácticos, ya sea porque las relaciones con los demás capítulos son demasiado intensas o porque la complejidad del análisis general resultante es escasamente operativa. Por otro lado, identificar las políticas con cada una de las unidades organizativas implicadas (considerar, por ejemplo, la política agrícola como el conjunto de las funciones desarrolladas por la Consejería de Agricultura) comportaría el riesgo de eliminar las diferencias existentes entre actividades distintas entre sí.

En general, la elección analítica más correcta será la de agregar entre sí las acciones tendentes a afrontar un problema (es decir, una necesidad o una demanda insatisfecha) común. En este sentido, la primera operación de la Administración pública consistirá en relacionar las leyes y los programas en que se basan las distintas actividades administrativas con los recursos (económicos y financieros y también organizativos) asignados, tratando de detectar los problemas para cuya solución se destinan unas y otros. No se trata de un ejercicio fácil, si bien podemos contar con una base informativa (las leyes y los programas, por un lado; los recursos financieros y organizativos, por otro) habitualmente disponible en las Administraciones públicas.

Una vez identificadas las unidades de análisis (en nuestro caso las políticas públicas) la situación se invierte y mientras la actividad analítica aparece relativamente fácil, la disponibilidad de informaciones 
constituye el problema principal, siendo a menudo necesaria la organización de un sistema informativo especializado. Los datos necesarios para construir el sistema de indicadores/señales de alarmà antes mencionado son esencialmente de tres tipos.

Ante todo necesitamos datos utilizables sobre los productos de la actividad administrativa, un primer tipo de información del que se carece habitualmente. Por muy paradógico que nos pueda parecer, a menudo la Administración no sabe lo que produce (cuántos $\mathrm{km} /$ alumno se recorren en el transporte escolar, cuántas comidas se distribuyen efectivamente en las escuelas, cuántos niños se hallan inscritos y cuántos asisten regularmente a una guardería o a una escuela materna, cuántas horas de asistencia domiciliaria se destinan a cada anciano, cuántos libros se consultan o se prestan en las bibliotecas públicas, etc.), todavía más si tenemos en cuenta que para algunas funciones y materias dicha operación plantea problemas objetivos. Por tanto, el primer paso necesario para poder hablar de control de gestión consiste en crear un mecanismo de recogida de dichas informaciones e, incluso antes, reflexionar sobre los datos susceptibles de representar con mayor exactitud las varias dimensiones cuantitativas y cualitativas del servicio.

El segundo bloque de datos necesarios se refiere a los costes directa e indirectamente imputables a la producción y distribución de los productos de las políticas. A este respecto cabe subrayar que, sobre todo en una administración regional con funciones de dirección y programación de servicios generalmente no gestionados de forma directa, deberemos tener en cuenta el conjunto de los recursos financieros utilizados, con independencia del hecho de que éstos procedan de sujetos distintos de la administración regional (entes locales, familias, tarifas, etc.). Por tanto, también en este caso la actividad de recogida de la información plantea problemas teóricos y prácticos de no fácil solución.

El tercer y último bloque de informaciones necesarias para establecer una actividad de valoración sistemática es el que presenta actualmente mayores carencias, rayando incluso la total ausencia. En efecto, ¿qué indicadores podemos utilizar para representar la demanda o la necesidad que debería satisfacer la política o el objetivo que debería alcanzar?, ¿cómo medir, por ejemplo, la «necesidad de conservación» implícita en la actividad museística?, o también, ¿cuál es el verdadero objetivo de la formación profesional de base?: facilitar la inserción de la población juvenil en el mercado del trabajo o garantizar también una educación posterior a la de la escuela obligatoria, y ¿cómo medir si se ha alcanzado una u otra? Las preguntas podrían continuar haciéndose cada vez más difíciles. Sin embargo, no hay que subvalorar la importancia de este tipo de especulaciones. La validez de un sistema de evaluación depende, en efecto, más de su capacidad de formu- 
lar las preguntas adecuadas que de su habilidad para ofrecer las respuestas justas. Llegados a este punto y relacionando, por un lado, los productos con las necesidades y, por el otro, con los costes, dispondremos de una serie de indicadores de eficacia y eficiencia, respectivamente, que constituyen precisamente el sistema de señales de alarma antes comentado. A partir de aquí podemos tratar de averiguar, en el seno del proceso de producción y distribución de bienes y servicios y habida cuenta de la disponibilidad de la Administración pública, en general, y de la regional, en particular, qué factores pueden servir para determinar la mejor satisfacción de las necesidades y/o la menor relación entre el coste y la unidad de producto. Dicho esquema conceptual y el sistema informativo que éste requiere pueden parecer demasiado complejos e incluso inútiles. En particular, cuando se sugiere a los responsables de las administraciones de gasto - con argumentos que no podemos desarrollar aquí- la exigencia de construir un sistema de control de gestión se producen dos reacciones típicas implícitamente contradictorias entre sí. La primera, ya apuntada, se refiere a la heterogeneidad extrema de las condiciones de producción del servicio, lo que impediría cualquier generalización, más aún si los factores que determinan la eficacia y la eficiencia son tan numerosos que cada caso constituye una excepción. A menudo no sirve de nada objetar la tradición de análisis e investigación sobre estos temas y que, dada por supuesta la imposibilidad de alcanzar una precisión absolu$\mathrm{ta}$, es posible ofrecer una idea general con respecto al estado de actuación de la política pública que no se base, pura y simplemente, en las impresiones, siempre interesantes, de los responsables de la política. La segunda respuesta, contradictoria con la primera, afirma la inutilidad del ejercicio puesto que el responsable conocía muy bien qué pasos son necesarios y qué medidas deben adoptarse para mejorar la eficacia y la eficiencia que se consideran no medibles. Generalmente, las respuestas se refieren a la racionalización de los servicios, su concentración en unidades productivas de mayor dimensión, al objeto de aprovechar las economías de escala, y la reducción de los efectos externos, al margen de cualquier análisis relativo a su capacidad real de mejorar la eficacia y la eficiencia.

Como es sabido, este tipo de reformismo institucional y organizativo, que inspiró en muchos países las reorganizaciones de los años sesenta y setenta, provocó en su momento grandes esperanzas y fuertes desilusiones. La introducción de un control de gestión basado en el análisis de las políticas públicas puede ser un instrumento importante para compensar unas y otras. 


\section{PROBLEMAS ORGANIZATIVOS DEL CONTROL DE GESTION}

Para concluir estas notas nos resta sólo afrontar brevemente algunos aspectos más exquisitamente organizativos relativos a la introducción del control de gestión en la Administración regional.

El primero se refiere al carácter centralizado o descentralizado que debe revestir dicha actividad. Nuestro razonamiento debe partir del siguiente punto: cuando hablamos de políticas públicas en general nos referimos a la actividad de los distintos sectores en que se articula una organización compleja como la región. Hablamos, por tanto, de política sanitaria, y en el seno de ésta, de política de asistencia psiquiátrica o de política industrial, de política de formación profesional, etc. Para este tipo de actividades presuponemos la utilidad de introducir el control de gestión, entendido como comprobación de demandas, productos, costes de la acción pública. En realidad, también podríamos imaginar un control de eficiencia y eficacia de las llamadas políticas «horizontales» (personal, presupuesto, programación, etc.). En este caso, sin embargo, los problemas serían mucho más complejos y, por tanto, vale más concentrar nuestra atención en las políticas sectoriales. En este sentido, la primera pregunta que conviene plantear es la siguiente: ¿esta comprobación de eficacia y eficiencia debe realizarse de forma central para toda la Administración regional o bien en cada uno de los sectores? Desde un cierto punto de vista parecería más natural escoger la segunda alternativa: después de todo parece más natural que el control se halle en manos de los responsables de la política, más aún si tenemos en cuenta que la construcción de un sistema centralizado plantea difíciles problemas de homogeneización. Sin embargo, conviene recordar el carácter de revolución cultural implícito en la introducción de la evaluación sistemática de las acciones públicas. Y, desde que el mundo es mundo, las revoluciones las han realizado y dirigido pequeñas minorías iluminadas que han presionado hacia metas que difícilmente podrían ser alcanzadas a través de las tendencias espontáneas de evolución del sistema. Conviene no olvidar, en efecto, el carácter controvertido que implica dicho ejercicio: la elección de las variables que pretendemos controlar y de los indicadores capaces de medirlas (y, a un nivel distinto, de los modelos de análisis) puede ocultar también importantes problemas político-administrativos. En una situación en la que los sectores compiten entre sí para la asignación de recursos escasos por definición, atribuir a cada uno de éstos la responsabilidad de suministrar informaciones convincentes sobre el grado de eficiencia y eficacia de su propio servicio puede desencadenar además mecanismos perversos susceptibles de afectar a la credibilidad de la operación. Por 
estas razones, a las que se añade la necesidad de construir un sistema informativo abierto y flexible, aunque dotado de coherencia y homogeneidad internas, la opción de constituir un núcleo central responsable de activar el control parece, por tanto, claramente preferible.

El segundo problema, de carácter organizativo, se refiere a la decisión de establecer dicho núcleo en el Parlamento o en el Ejecutivo. Aquí no caben razonamientos demasiado complejos; una vez definida la actividad de control como una función eminentemente directiva, su centro de imputación no puede ser sino el Ejecutivo, es decir, el órgano responsable en primer grado de la aplicación de las políticas regionales. Se trata de una decisión fundamental que se deriva coherentemente de las premisas metodológicas de nuestro razonamiento y que no puede ser atacada si se acepta el planteamiento seguido. Sin embargo, el hecho de atribuir esta responsabilidad al Ejecutivo no significa dejar al margen al Parlamento; al contrario, las relaciones han de ser frecuentes, desarrollándose en ambas direcciones. El Parlamento debe disponer, en efecto, del derecho de interrogación del servicio del control de gestión en lo que respecta al estado de ejecución efectiva de las políticas decididas mediante leyes regionales. Y ello no sólo para garantizar a los parlamentarios el acceso a los productos del control (el informe general sobre la gestión regional y las eventuales elaboraciones en materia de cada política), sino también para suministrar inputs relevantes al citado control (por ejemplo, solicitar estudios informativos y valoraciones sobre aspectos particulares de eficiencia, eficacia y equidad de la acción pública). La oportunidad de asegurar este derecho de acceso se desprende de la consideración del modo, a menudo consensual, con que se desarrolla la actividad legislativa y de dirección del órgano directivo; la búsqueda de compromisos aceptables entre la mayoría y la minoría, y una disminución general del grado de conflictividad sobre cada una de las políticas serán más efectivos en la medida en que el Parlamento disponga de los medios necesarios para comprobar que las decisiones adoptadas en su seno no sufren ulteriormente modificaciones a través de la actividad diaria de las Consejerías. Este razonamiento es válido para la mayoría, pero también, y ante todo, para las relaciones mayoría/oposición. Este aspecto debe valorarse seriamente, ya que contribuye a aumentar la autonomía y la autoridad del control frente al resto de la administración regional. Sin embargo, las relaciones deben funcionar también en sentido contrario: un servicio de control de gestión, competente y eficiente, también debe hallarse en condiciones de poner en guardia al órgano legislativo con respecto a las consecuencias que la persecución de determinados objetivos, la elección de determinados procedimientos o la movilización de determinados recursos puede comportar en la actividad diaria. Este parece el modo más correcto y eficaz de afrontar la cuestión, muy deba- 
tida en el pasado, de la factibilidad de las leyes, sabiendo, en cualquier caso, que las competencias profesionales para desarrollar este tipo de actividad son realmente escasas, de modo que plantear duplicidades, además de ser poco eficiente desde el punto de vista económico, aparece también improbable desde el punto de vista organizativo. Si la evaluación sistemática consigue mejorar la calidad de la producción legislativa regional deberemos felicitarnos sinceramente.

La segunda cuestión se refiere, lógicamente, a la ubicación del núcleo dentro del Ejecutivo. Existen tres opciones posibles: la Presidencia; competente en materia de personal y organización regional; el Departamento responsable del presupuesto y la programación. Esta última alternativa debe descartarse por distintos motivos, implícita y explícitamente enunciados en las páginas precedentes. Una actividad de programación debe orientarse esencialmente hacia el futuro, mientras que el análisis de las políticas regionales, analizado bajo la forma de control de gestión, debe basarse en estudios retrospectivos. En este sentido podemos, y debemos, aprender del pasado, prescindiendo incluso del hecho de que en el futuro los objetivos, los instrumentos, o ambos a la vez, están destinados a cambiar. La responsabilidad de remediar la falta de memoria de la Administración no puede asignarse a una unidad organizativa cuya tarea principal consiste en prever el futuro adelantando las opciones necesarias para dirigirlo. Por razones todavía más evidentes, dicha responsabilidad no puede corresponder a los responsables de la organización y del personal; como se ha indicado, las políticas cuya eficiencia y eficacia debemos analizar escapan, por lo general, a la organización regional en el sentido estricto y requieren relaciones con otros sujetos político-administrativos. Sería un error conceptual, además de un mecanismo sustancialmente inútil, confundir ambos niveles y reducir el control a la comprobación de la funcionalidad de la organización burocrática regional. Además, y esto vale para las dos opciones hasta ahora examinadas, hay un aspecto político que no podemos descuidar. Situar la función de valoración de la eficiencia y de la eficacia del conjunto de la Administración bajo la responsabilidad de un único Departamento comporta el riesgo de determinar (sobre todo, aunque no sólo, en el caso de los gobiernos de coalición) reacciones políticas perversas en la medida en que los responsables políticos de las Administraciones de gasto no se hallan dispuestos a aceptar espontáneamente un papel de este tipo. Por consiguiente, la elección más correcta parece ser la de situar esta actividad bajo la responsabilidad del presidente como corresponde a su superior papel, que representa, o debería representar, el centro de síntesis general y de unidad de la orientación político-administrativa regional. Además de ser más coherente desde el punto de vista institucional (también en función de las relaciones con el Legislativo antes comentadas), dicha acción pre- 
senta menores costos organizativos frente a unos beneficios máximos para la funcionalidad del ejercicio.

El último tẹma se plantea a un nivel parcialmente distinto, por lo que requiere una atención especial. La actividad de control de gestión no es y no debe ser una actividad de estudio e investigación y tampo$\mathrm{co}$, únicamente, una actividad informativa. Para las exigencias de profundización teórica y analítica existe la posibilidad de encargar informes externos, mientras que la recogida de información puede basarse en un instrumento general, como un Sistema Informativo (si bien deberemos considerar atentamente los datos específicos necesarios para la valoración de las políticas). La actividad de control de gestión se justifica en base a una exigencia de dirección de la Administración, para la cual la información y la organización de éstas en modelos de análisis más apropiados constituye una condición necesaria, pero no suficiente. El control de gestión no es una tarea para profesores universitarios o para expertos informáticos; requiere, al contrario, administradores expertos y motivados, dispuestos a participar en la formulación de opciones relativas a una pluralidad de sectores y de problemáticas. Dicha función se plantea como uno de los instrumentos utilizables para restituir flexibilidad a una Administración carente de ella desde hace tiempo, si es que alguna vez la ha tenido. Representa algo esencial para modificar las situaciones negativas que se han venido cristalizando a lo largo del tiempo, introduciendo y haciendo circular la innovación organizativa con objeto de mejorar todo lo mejorable. Todos estos objetivos requieren instrumentos operativos flexibles y no rígidos. Ya hemos prevenido anteriormente contra el error de construir un sistema vinculante de indicadores y contra los peligros de circuitos perversos que ello comporta. Es el momento, ahora, de romper una lanza a favor del reforzamiento de una actitud favorable a la solución de los problemas en el seno de la Administración. Un sistema de señales de alarma no sirve para activar sistemas sancionádores y autoritarios. Sirve, al contrario, para poner a las unidades organizativas responsables de cada servicio en condiciones de detectar lo que no está funcionando correctamente y para estudiar, junto con los operadores de base, los medios adecuados para eliminar los obstáculos, aprovechando las oportunidades existentes. En este sentido, la constitución en cada Administración sectorial de unidades de inspección pequeñas y ágiles, capaces de recibir del control de gestión las indicaciones o informaciones necesarias y de personarse in situ para tratar de resolver los problemas más urgentes, parece la principal condición de eficacia ligada a la introducción de la valoración. Sin duda nos hallamos muy lejos de las grandes estructuras de planificación racional de los años sesenta y setenta. A pesar de ello (o quizá por este mismo motivo), los resultados podrán ser más positivos, incluso a medio y corto plazo, si el objetivo consiste en construir una Administración pública responsable y eficaz.

Traducción de Francesc Morata 
DA-1990-1991, núms. 224-225. BRUNO DENTE. Análisis de las políticas públicas en la práctic...

DA-1990-1991, núms. 224-225. BRUNO DENTE. Análisis de las políticas públicas en la práctic... 